\title{
On Solutions of Linear Functional Systems and Factorization of Laurent-Ore Modules
}

\author{
Ziming Li \\ Key Lab of Mathematics-Mechanization \\ Academy of Mathematics and System Sciences \\ Zhong Guan Cun, Beijing 100080, China \\ zmli@mmrc.iss.ac.cn \\ Min $\mathrm{Wu}^{1)}$ \\ Software Engineering Institute \\ East China Normal University \\ North Zhangshan Road, Shanghai 200062, China \\ mwu@sei.ecnu.edu.cn
}

\begin{abstract}
We summarize some recent results on partial linear functional systems. By associating a finite-dimensional linear functional system to a Laurent-Ore module, Picard-Vessiot extensions are generalized from linear ordinary differential (difference) equations to finite-dimensional linear functional systems. A generalized Beke's method is also presented for factoring Laurent-Ore modules and it will allow us to find all "subsystems" whose solution spaces are contained in that of a given linear functional system.
\end{abstract}

\section{Introduction}

This paper provides a survey of the work by M. Bronstein and the authors in a FranceSino Scientific Cooperation Project ${ }^{2)}$ from 2002 to 2005. Our project concerns finitedimensional linear functional systems, and its outcome includes: a generalization of PicardVessiot extensions of linear ordinary differential (difference) equations, the notion of modules of formal solutions, algorithms for computing the dimension of solution spaces and for reducing linear functional systems to fully integrable ones, and generalizations of Beke's factorization algorithm and of the eigenring method. The emphasis of this paper is on descriptions of these results. Precise references are given for proofs and technical details.

A (partial) linear functional system consists of linear partial differential, shift, and $q$-shift operators, or any mixture thereof. By a finite-dimensional linear functional system, or a $\partial$ finite system for short, we mean a linear functional system whose module of formal solutions has finite dimension (see Definition 4.5). Intuitively, a system is $\partial$-finite if and only if its

\footnotetext{
1) Partially supported by a KLMM Open Project Funding KLMM-0611.

${ }^{2)}$ This joint project is supported in part by the French Government Scholarship (BGF no. 2002915), an INRIA-CAFÉ Project Funding and two National Key Projects of China (no. 1998030600, 2004CB31830).
} 
solution space has finite dimension. The following is an example:

$$
\begin{cases}P^{\prime \prime}(x, k)-\frac{2 x}{1-x^{2}} P^{\prime}(x, k)+\frac{k(k+1)}{1-x^{2}} P(x, k) & =0 \\ P(x, k+2)-\frac{(2 k+3) x}{k+2} P(x, k+1)+\frac{k+1}{k+2} P(x, k) & =0 .\end{cases}
$$

The sequence of the Legendre polynomials $\{P(x, k)\}_{k=1}^{\infty}$ is a solution of (1) with the initial conditions $\left\{P(0,0)=0, P^{\prime}(0,0)=0, P(0,1)=0, P^{\prime}(0,1)=1\right\}$.

Given a linear functional system $L$, we are interested in the following questions: (i) Does $L$ have a nonzero solution? (ii) Is there a ring containing "all" the solutions of $L$ ? (iii) How does one compute the dimension of the solution space of $L$ ? (iv) How does one find (if it exists) a "subsystem" whose solution space is properly contained in that of $L$ ? (v) Determine whether the solution space of $L$ can be written as a direct sum of those of its subsystems?

Our work is intended for answering these questions algorithmically for $\partial$-finite systems. In terms of modules of formal solutions (Definition 4.5) and Picard-Vessiot extensions (Definition 4.8), the above questions translate respectively to: (i) Is a module $M$ of formal solutions trivial? (ii) Does there exist a Picard-Vessiot extension for a given system? (see Section 4.) (iii) How does one compute the dimension of $M$ ? (see Section 5.) (iv) How does one find a nontrivial submodule of $M$ ? (see Section 6.) (v) Is $M$ decomposable? (see Section 6.)

Many of the results in this paper are straightforward generalizations of their counterparts of linear ordinary differential or difference equations. These generalizations are however necessary in view of their wider applicability and the complications caused by the appearance of several differential and difference operators.

Throughout the paper, rings are not necessarily commutative and have arbitrary characteristic. Ideals, modules and vector spaces are all left ones. Fields are always assumed to be commutative. Denote by $R^{p \times q}$ the set of all $p \times q$ matrices with entries in a ring $R$, and by $\mathbf{e}_{i n}$, for $1 \leq i \leq n$, the unit vector in $R^{1 \times n}$ with 1 in the $i$ th position and 0 elsewhere. The notation " $\cong_{R}$ " means "isomorphic as $R$-modules". We use $(\cdot)^{\tau}$ to denote the transpose of a vector or matrix, and $\mathbf{1}_{n}$ to denote the identity matrix of size $n$. Vectors are represented by the boldfaced letters $\mathbf{u}, \mathbf{v}, \mathbf{w}$ etc. Vectors of unknowns are denoted $\mathbf{x}, \mathbf{y}, \mathbf{z}$, etc. The symbol $\mathbb{C}$ denotes the field of complex numbers.

The paper is organized as follows. In Section 2., we present some preliminaries and define the notion of linear functional systems. In Section 3., we construct Picard-Vessiot extensions for fully integrable systems, which are a common special case of $\partial$-finite systems. In Section 4., modules of formal solutions are defined and Picard-Vessiot extensions are generalized for $\partial$-finite systems. In Section 5., we present some techniques for computing linear dimension of a linear functional system. In Section 6., we generalize Beke's algorithm and the eigenring approach to factor Laurent-Ore modules. Concluding remarks are made in Section 7.

\section{Preliminaries}

Let $R$ be a ring and $\Delta$ be a finite set of commuting maps from $R$ to itself. A map in $\Delta$ is assumed to be either a derivation or an automorphism. Recall that a derivation $\delta$ is an 
additive map satisfying the multiplicative rule $\delta(a b)=a \delta(b)+\delta(a) b$ for all $a, b \in R$. The pair $(R, \Delta)$ is called a $\Delta$-ring, and it is a $\Delta$-field when $R$ is a field.

For a derivation $\delta \in \Delta$, an element $c$ of $R$ is called a constant with respect to $\delta$ if $\delta(c)=0$. For an automorphism $\sigma \in \Delta, c$ is called a constant with respect to $\sigma$ if $\sigma(c)=c$. An element $c$ of $R$ is called a constant if it is a constant with respect to all maps in $\Delta$. The set of constants of $R$, denoted by $C_{R}$, is a subring. The $\operatorname{ring} C_{R}$ is a subfield if $R$ is a field.

Let $(F, \Delta)$ be a $\Delta$-field. By reordering the indices, we can always assume that $\Delta=$ $\left\{\delta_{1}, \ldots, \delta_{\ell}, \sigma_{\ell+1}, \ldots, \sigma_{m}\right\}$ for some $\ell \geq 0$, where the $\delta_{i}$ 's are derivation operators on $F$ and the $\sigma_{j}$ 's are automorphisms of $F$. The Ore algebra ([8]) over $F$ is the polynomial ring $\mathcal{S}:=$ $F\left[\partial_{1}, \ldots, \partial_{m}\right]$ in $\partial_{i}$ with the usual addition and a multiplication as follows:

$$
\partial_{i} \partial_{j}=\partial_{j} \partial_{i}, \quad \partial_{s} a=a \partial_{s}+\delta_{s}(a), \quad \partial_{t} a=\sigma_{t}(a) \partial_{t},
$$

for any $1 \leq i, j \leq m, 1 \leq s \leq \ell, \ell<t \leq m$ and $a \in F$.

Remark that $\partial_{i}(a)$, where $a$ is an element of a $\Delta$-ring, is meant to be $\delta_{i}(a)$ if $\partial_{i}$ is associated to a derivation operator $\delta_{i}$, and to be $\sigma_{i}(a)$ if $\partial_{i}$ is associated to an automorphism $\sigma_{i}$; while $\partial_{i} a$, where $a$ is an element of the Ore algebra $\mathcal{S}$, means the product of $\partial_{i}$ and $a$.

Definition 2.1 Let $(F, \Delta)$ be a $\Delta$-field. A linear functional system over $F$ is a system of the form $A(\mathbf{z})=0$ where $A$ is a $p \times q$ matrix with entries in the Ore algebra $\mathcal{S}$ and $\mathbf{z}$ is a column vector of $q$ unknowns.

Example 2.2 The system (1), satisfied by the Legendre polynomials, can be rewritten as $A(z)=0$ where $A=\left(\partial_{x}^{2}-\frac{2 x}{1-x^{2}} \partial_{x}+\frac{k(k+1)}{1-x^{2}}, \partial_{k}^{2}-\frac{(2 k+3) x}{k+2} \partial_{k}+\frac{k+1}{k+2}\right)^{\tau}$, with $\partial_{x}$ the differentiation with respect to $x$ and $\partial_{k}$ the shift operator with respect to $k$.

Let $F$ be a $\Delta$-field. A commutative $\operatorname{ring} R$ containing $F$ is called a $\Delta$-extension of $F$ if all the maps in $\Delta$ can be extended to $R$ in such a way that all derivations (resp. automorphisms) of $F$ become derivations (resp. automorphisms) of $R$ and the extended maps commute pairwise.

By a solution of a linear functional system $A(\mathbf{z})=0$ over $F$, we mean a vector $\left(s_{1}, \ldots, s_{q}\right)^{\tau}$ over some $\Delta$-extension of $F$ such that $A\left(s_{1}, \ldots, s_{q}\right)^{\tau}=0$, i.e., the application of the matrix $A$ to the vector is zero.

\section{Fully integrable systems}

A common special case of linear functional systems consists of fully integrable systems, which are of the form $\left\{\partial_{i}(\mathbf{z})=A_{i} \mathbf{z}\right\}_{1 \leq i \leq m}$ and correspond to the linear functional system $A(\mathbf{z})=0$ where the matrix $A$ is given by the stacking of blocks of the form $\left(\partial_{i} \cdot \mathbf{1}_{n}-A_{i}\right)$. Fully integrable systems are of interest to our study, since to every $\partial$-finite system, we can associate a fully integrable system whose solution space is isomorphic to that of the original system (see Section 4.3.).

Definition 3.1 A system of the form

$$
\delta_{i}(\mathbf{z})=A_{i} \mathbf{z}, \quad 1 \leq i \leq \ell, \quad \sigma_{i}(\mathbf{z})=A_{i} \mathbf{z}, \quad \ell+1 \leq i \leq m,
$$


where $A_{i} \in F^{n \times n}$ and $\mathbf{z}$ is a column vector of $n$ unknowns, is called an integrable system of size $n$ over $F$ if the following compatibility conditions are satisfied:

$$
\begin{aligned}
\delta_{i}\left(A_{j}\right) & =\delta_{j}\left(A_{i}\right), & & 1 \leq i<j \leq \ell, \\
\sigma_{i}\left(A_{j}\right) A_{i} & =\sigma_{j}\left(A_{i}\right) A_{j}, & & \ell<i<j \leq m, \\
\sigma_{j}\left(A_{i}\right) A_{j} & =A_{i} A_{j}+\delta_{i}\left(A_{j}\right), & & 1 \leq i \leq \ell<j \leq m .
\end{aligned}
$$

The integrable system (2) is said to be fully integrable if the matrices $A_{\ell+1}, \ldots, A_{m}$ are invertible.

Using Ore algebra notation, we write $\left\{\partial_{i}(\mathbf{z})=A_{i} \mathbf{z}\right\}_{1 \leq i \leq m}$ for the system (2) where the action of $\partial_{i}$ is again meant to be $\delta_{i}$ for $i \leq \ell$ and to be $\sigma_{i}$ for $i>\ell$. Observe that the conditions (3) are derived from the condition $\partial_{i}\left(\partial_{j}(\mathbf{z})\right)=\partial_{j}\left(\partial_{i}(\mathbf{z})\right)$ and are exactly the matrix-analogues of the compatibility conditions for first-order scalar equations in [11]. For a linear ordinary difference equation, we often assume that its trailing coefficient is nonzero, while, for a first-order matrix difference equation, we assume that its matrix is invertible. These assumptions lead to the condition on invertibility of $A_{\ell+1}, \ldots, A_{m}$ in Definition 3.1.

Example 3.2 Let $F=\mathbb{C}(x, k), \delta_{x}$ be the differentiation with respect to $x$ and $\sigma_{k}$ the shift operator with respect to $k$. Then $\mathcal{A}:\left\{\delta_{x}(\mathbf{z})=A_{x} \mathbf{z}, \sigma_{k}(\mathbf{z})=A_{k} \mathbf{z}\right\}$ is a fully integrable system where

$$
A_{x}=\left(\begin{array}{cc}
\frac{x^{2}-k x-k}{x(x-k)(x-1)} & \frac{x^{2}-k x+3 k-2 x}{k x(x-k)(x-1)} \\
\frac{k\left(k x+x-x^{2}-2 k\right)}{(x-k)(x-1)} & \frac{x^{3}+x^{2}-k x^{2}-2 x+2 k}{x(x-k)(x-1)}
\end{array}\right) \text { and } A_{k}=\left(\begin{array}{cc}
\frac{k+1+k x^{2}-x k^{2}-x}{(x-k)(x-1)} & -\frac{k+1+k x-k^{2}-x}{k(x-k)(x-1)} \\
\frac{x(k+1)\left(k+1+k x-k^{2}-x\right)}{(x-k)(x-1)} & \frac{(k+1)\left(x^{2}-2 k x-x+k^{2}\right)}{k(x-k)(x-1)}
\end{array}\right) .
$$

In what follows, we generalize fundamental matrices and Picard-Vessiot extensions of linear ordinary differential (difference) equations to fully integrable systems.

A square matrix with entries in a commutative ring is said to be invertible if its determinant is a unit in that ring.

Let $F$ be a $\Delta$-field and $\left\{\partial_{i}(\mathbf{z})=A_{i} \mathbf{z}\right\}_{1 \leq i \leq m}$ be a fully integrable system of size $n$ over $F$. We define

Definition 3.3 An $n \times n$ matrix $U$ with entries in a $\Delta$-extension of $F$ is a fundamental matrix for the system $\left\{\partial_{i}(\mathbf{z})=A_{i} \mathbf{z}\right\}_{1 \leq i \leq m}$ if $U$ is invertible and $\partial_{i}(U)=A_{i} U$ for each $i$, i.e., each column of $U$ is a solution of the system.

A two-sided ideal $I$ of a commutative $\Delta$-ring $R$ is said to be invariant if $\delta_{i}(I) \subset I$ for $i \leq \ell$ and $\sigma_{j}(I) \subset I$ for $j>\ell$. The ring $R$ is said to be simple if its only invariant ideals are (0) and $R$.

Definition 3.4 A Picard-Vessiot ring for a fully integrable system is a (commutative) ring $E$ such that:

(i) $E$ is a simple $\Delta$-extension of $F$.

(ii) There exists some fundamental matrix $U$ with entries in $E$ for the system such that $E$ is generated by the entries of $U$ and $\operatorname{det}(U)^{-1}$ over $F$. 
Definitions 3.3 and 3.4 are natural generalizations of their analogues in the purely differential case $[20$, (pages 12, 415)] and the ordinary difference case [19, (Errata)].

The existence of fundamental matrices and Picard-Vessiot extensions for fully integrable systems is stated in the following

Theorem 3.5 Every fully integrable system over $F$ has a Picard-Vessiot ring E. If $F$ has characteristic 0 and $C_{F}$ is algebraically closed, then $C_{E}=C_{F}$. Furthermore, that extension is minimal, meaning that no proper subring of E satisfies both conditions in Definition 3.4.

A detailed proof of the above theorem is found in [6].

Consequently, if $F$ has characteristic zero and an algebraically closed field of constants, then all the solutions of a fully integrable system in its Picard-Vessiot ring form a $C_{F}$-vector space whose dimension equals the size of the system.

We now present two examples for Picard-Vessiot extensions. The reader is referred to $[25, \S 2.2]$ for detailed verifications.

Example 3.6 Consider the fully integrable system of size one:

$$
\partial_{i}(z)=a_{i} z \quad \text { where } a_{i} \in F \text { and } i=1, \ldots, m .
$$

This is an extension of Example 1.19 in [20].

Let $E=F\left[T, T^{-1}\right]$ be the $\Delta$-extension such that $\delta_{i}(T)=a_{i} T$ and $\sigma_{j}(T)=a_{j} T$ for $i \leq \ell$ and $j>\ell$. Then $E$ is a Picard-Vessiot ring of the given system if there does not exist an integer $k>0$ and a nonzero $r \in F$ such that $\delta_{i}(r)=k a_{i} r$ for $i \leq \ell$ and $\sigma_{j}(r)=a_{j}^{k} r$ for $j>\ell$. Otherwise, assume that the integer $k>0$ is minimal so that there exists a nonzero $r \in F$ satisfying $\delta_{i}(r)=k a_{i} r$ for $i \leq \ell$ and $\sigma_{j}(r)=a_{j}^{k} r$ for $j>\ell$. Then $E /\left(T^{k}-r\right)$ is a PicardVessiot ring of the given system.

Example 3.7 Consider the system $\mathcal{A}$ in Example 3.2. Note that the change of variable ${ }^{3)}$ $\mathbf{z}=$ My where

$$
M=\left(\begin{array}{cc}
\frac{x-k}{x} & x^{2} \\
(x-k) k & x^{2} k
\end{array}\right)
$$

transforms $\mathcal{A}$ into another fully integrable system $\mathcal{B}:\left\{\delta_{x}(\mathbf{y})=B_{x} \mathbf{y}, \sigma_{k}(\mathbf{y})=B_{k} \mathbf{y}\right\}$ with $B_{x}=\left(\begin{array}{ll}1 & 0 \\ 0 & 0\end{array}\right)$ and $B_{k}=\left(\begin{array}{ll}1 & 0 \\ 0 & k\end{array}\right)$. It suffices to find a Picard-Vessiot ring of $\mathcal{B}$. We get that $V=\left(\begin{array}{cc}e^{x} & 0 \\ 0 & \Gamma(k)\end{array}\right)$ is a fundamental matrix for $\mathcal{B}$, and thus $M V$ is for $\mathcal{A}$. Moreover, $F\left[e^{x}, \Gamma(k), e^{-x}, \Gamma(k)^{-1}\right]$ is a Picard-Vessiot extension for $\mathcal{A}$.

\section{4. $\partial$-finite systems}

In this section, we first discuss generic solutions of linear algebraic equations over arbitrary rings, then introduce the notions of Laurent-Ore algebras and modules of formal solutions. These two notions allow us to generalize the results in Section 3. to $\partial$-finite systems.

\footnotetext{
${ }^{3)}$ which can be found, for example, by computing the hyperexponential solutions of the system $([11,25])$
} 


\subsection{Generic solutions of linear algebraic equations over rings}

Let $R$ be an arbitrary ring. Denote by $Z(R)$ the center of $R$, i.e. the set of all elements that commute with every element in $R$. Then $Z(R)$ is a subring of $R$. Consider a $p \times q$ matrix $A=\left(a_{i j}\right)$ with entries in $R$. For any $R$-module $N$, we can associate to $A$ a $Z(R)$-linear map $\lambda: N^{q} \rightarrow N^{p}$ given by

$$
\xi:=\left(\xi_{1}, \ldots, \xi_{q}\right)^{\tau} \mapsto A \xi=\left(\sum_{j=1}^{q} a_{1 j} \xi_{j}, \ldots, \sum_{j=1}^{q} a_{p j} \xi_{j}\right)^{\tau} .
$$

We therefore say that $\xi \in N^{q}$ is a solution "in $N$ " of the system $A(\mathbf{z})=0$ if $\lambda(\xi)=0$, and write $\operatorname{sol}_{N}(A(\mathbf{z})=0)$ for the set of all solutions in $N$. Clearly, $\operatorname{sol}_{N}(A(\mathbf{z})=0)$ is a $Z(R)$-module. Note that $\lambda$ is in general not $R$-linear since $R$ is noncommutative.

As in the case of $\mathcal{D}$-modules [16], we can associate to $A \in R^{p \times q}$ an $R$-module as follows: $A$ induces the $R$-linear map $\rho: R^{1 \times p} \rightarrow R^{1 \times q}$ given by $\left(r_{1}, \ldots, r_{p}\right) \mapsto\left(r_{1}, \ldots, r_{p}\right) A$. Let $M=$ $R^{1 \times q} /\left(R^{1 \times p} A\right)$, which is the quotient of $R^{1 \times q}$ by the image of the map $\rho$. We call $M$ the $R$ cokernel of $A$ and denote it by $\operatorname{coker}_{R}(A)$. Clearly, $\operatorname{coker}_{R}(A)$ is an $R$-module. Let $\mathbf{e}_{1 p}, \ldots, \mathbf{e}_{p p}$ and $\mathbf{e}_{1 q}, \ldots, \mathbf{e}_{q q}$ be the canonical bases of $R^{1 \times p}$ and $R^{1 \times q}$, respectively. Denote by $\pi$ the canonical map from $R^{1 \times q}$ to $\operatorname{coker}_{R}(A)$, and set $\mathbf{e}_{j}=\pi\left(\mathbf{e}_{j q}\right)$ for $1 \leq j \leq q$. Since $\pi$ is surjective, $M$ is generated by $\mathbf{e}_{1}, \ldots, \mathbf{e}_{q}$ over $R$. Note that $\rho\left(\mathbf{e}_{i p}\right)$ is the $i$-th row of $A$. Hence

$$
0=\pi\left(\rho\left(\mathbf{e}_{i p}\right)\right)=\pi\left(\sum_{j=1}^{q} a_{i j} \mathbf{e}_{j q}\right)=\sum_{j=1}^{q} a_{i j} \pi\left(\mathbf{e}_{j q}\right)=\sum_{j=1}^{q} a_{i j} \mathbf{e}_{j}, \quad \text { for } 1 \leq i \leq p,
$$

which implies that $\left(\mathbf{e}_{1}, \ldots, \mathbf{e}_{q}\right)^{\tau}$ is a solution of $A(\mathbf{z})=0$ in $M$.

Given two $R$-modules $N_{1}$ and $N_{2}$, denote by $\operatorname{Hom}_{R}\left(N_{1}, N_{2}\right)$ the set of all $R$-linear maps from $N_{1}$ to $N_{2}$. Clearly, $\operatorname{Hom}_{R}\left(N_{1}, N_{2}\right)$ is a $Z(R)$-module.

As illustrated by the following theorem, Proposition 1.1 of [16] remains true when $\mathcal{D}$ is replaced by an arbitrary ring $R$.

Theorem 4.1 Let $M=R^{1 \times q} /\left(R^{1 \times p} A\right)$. Then $\operatorname{Hom}_{R}(M, N)$ and $\operatorname{sol}_{N}(A(\mathbf{z})=0)$ are isomorphic as $Z(R)$-modules for any $R$-module $N$.

Remark 4.2 (i) The proof of Proposition 1.1 in [16] can be adapted to this theorem in a straightforward way (see [6]) and also, a slightly different but elementary proof is given in [25, Theorem 2.4.1].

(ii) The proof of Theorem 4.1 reveals that the vector $\mathbf{e}:=\left(\mathbf{e}_{1}, \ldots, \mathbf{e}_{q}\right)^{\tau} \in M^{q}$ specified above is a "generic" solution of the system $A(\mathbf{z})=0$ in the sense that any solution $\left(s_{1}, \ldots, s_{q}\right)^{\tau}$ of that system in $N$ is the image of $\mathbf{e}$ under the map in $\operatorname{Hom}_{R}(M, N)$ sending $\mathbf{e}_{i}$ to $s_{i}$.

\subsection{Laurent-Ore algebras}

Let $F$ be a $\Delta$-field and $\mathcal{S}=F\left[\partial_{1}, \ldots, \partial_{m}\right]$ be the corresponding Ore algebra. In the differential case, an $\mathcal{S}$-module is classically associated to a linear functional system [16, 20]. In the difference case, however, $\mathcal{S}$-modules may not have appropriate dimensions, as illustrated by the following counterexample. 
Example 4.3 Let $\sigma \neq \mathbf{1}$ be an automorphism of $F$ and $\mathcal{S}=F[\partial]$ be the corresponding Ore algebra. The equation $\partial(y)=0$ cannot have a fundamental matrix $(u)$ in any difference ring extension of $F$, for otherwise, $0=\partial(u)=\sigma(u)$, thus $u=0$. Therefore $\partial(y)=0$ has only trivial solution. However, the $\mathcal{S}$-module $\mathcal{S} / \mathcal{S} \partial$ has dimension one as an $F$-vector space.

In [19, page 56], modules over Laurent algebras are used instead to avoid the above problem. It is therefore natural to introduce the following extension of $\mathcal{S}$ : let $\theta_{\ell+1}, \ldots, \theta_{m}$ be indeterminates independent of the $\partial_{i}$. Since the $\sigma_{j}^{-1}$ are automorphisms of $F, \overline{\mathcal{S}}=$ $F\left[\partial_{1}, \ldots, \partial_{m}, \theta_{\ell+1}, \ldots, \theta_{m}\right]$ is also an Ore algebra in which the $\theta_{j}$ are associated to the $\sigma_{j}^{-1}$. Note that $\partial_{j} \theta_{j}$ is in the center of $\overline{\mathcal{S}}$, since

$$
\left(\partial_{j} \theta_{j}\right) a=\partial_{j} \sigma_{j}^{-1}(a) \theta_{j}=\sigma_{j}\left(\sigma_{j}^{-1}(a)\right) \partial_{j} \theta_{j}=a \partial_{j} \theta_{j}, \quad \text { for all } a \in F \text { and } j>\ell .
$$

Therefore the left ideal $T=\sum_{j=\ell+1}^{m} \overline{\mathcal{S}}\left(\partial_{j} \theta_{j}-1\right)$ is a two-sided ideal of $\overline{\mathcal{S}}$, and we call the factor ring $\mathcal{L}=\overline{\mathcal{S}} / T$ the Laurent-Ore algebra over $F$. Writing $\partial_{j}^{-1}$ for the image of $\theta_{j}$ in $\mathcal{L}$, we can write $\mathcal{L}$ (by convention) as $\mathcal{L}=F\left[\partial_{1}, \ldots, \partial_{m}, \partial_{\ell+1}^{-1}, \ldots, \partial_{m}^{-1}\right]$ and view it as an extension of $\mathcal{S}$. For linear ordinary difference equations, $\mathcal{L}=F\left[\sigma, \sigma^{-1}\right]$ is the algebra used in [19]. For linear partial difference equations with constant coefficients, $\mathcal{L}$ is the Laurent polynomial ring used in $[18,26]$.

Except for the purely differential case in which $\ell=0$, a Laurent-Ore algebra $\mathcal{L}=$ $F\left[\partial_{1}, \ldots, \partial_{m}, \partial_{\ell+1}^{-1}, \ldots, \partial_{m}^{-1}\right]$ is not an Ore algebra since $\partial_{j} \partial_{j}^{-1}=\partial_{j}^{-1} \partial_{j}=1$.

When revisiting Example 4.3 with Laurent-Ore algebras, we get that the left ideal generated by $\partial$ in $\mathcal{L}=F\left[\partial, \partial^{-1}\right]$ is $\mathcal{L}$, therefore the dimension of $\mathcal{L} /(\mathcal{L} \partial)$ over $F$, which is zero, equals that of the solution space of $\partial(y)=0$ in any difference ring extension.

In the sequel, a module over a Laurent-Ore algebra that is finite-dimensional over the ground field is called a Laurent-Ore module for short.

\subsection{Modules of formal solutions}

Let $F$ be a $\Delta$-field, and $\mathcal{S}$ and $\mathcal{L}$ be the corresponding Ore and Laurent-Ore algebras. Replacing $R$ with $\mathcal{L}$ in Theorem 4.1 yields

Theorem 4.4 Let $A \in \mathcal{S}^{p \times q}$ and $M=\operatorname{coker}_{\mathcal{L}}(A)$. Then $\operatorname{sol}_{N}(A(\mathbf{z})=0)$ and $\operatorname{Hom}_{\mathcal{L}}(M, N)$ are isomorphic as $C_{F}$-vector spaces for any $\mathcal{L}$-module $N$.

From Remark 4.2(ii) in which we replace arbitrary $\operatorname{ring} R$ with $\mathcal{L}, \operatorname{coker}_{\mathcal{L}}(A) \operatorname{describes}$ the properties of all the solutions of $A(\mathbf{z})=0$ "anywhere". This motivates us to define

Definition 4.5 Let $A \in \mathcal{S}^{p \times q}$. The $\mathcal{L}$-module $M=\mathcal{L}^{1 \times q} /\left(\mathcal{L}^{1 \times p} A\right)$ is called the module of formal solutions of the system $A(\mathbf{z})=0$. The dimension of $M$ as an $F$-vector space is called the linear dimension of the system. The system is said to be of finite linear dimension, or simply, $\partial$-finite, if $0<\operatorname{dim}_{F} M<+\infty$.

Note that we choose to exclude systems with $\operatorname{dim}_{F} M=0$ in the above definition since such system has only trivial solution in any $\mathcal{L}$-module, particularly, in any $\Delta$-extension of $F$.

Remark 4.6 For any $A \in \mathcal{S}^{p \times q}$, we can construct both its $\mathcal{S}$-cokernel coker ${ }_{\mathcal{S}}(A)$ and $\mathcal{L}$ cokernel $\operatorname{coker}_{\mathcal{L}}(A)$. Viewing $\mathcal{L}$ as a right $\mathcal{S}$-module and $\operatorname{coker}_{\mathcal{S}}(A)$ as a left $\mathcal{S}$-module, we can define the tensor product ([21]) $\mathcal{L} \otimes_{\mathcal{S}} \operatorname{coker}_{\mathcal{S}}(A)$, which is a right $\mathcal{S}$-module and a left $\mathcal{L}$ module. Lemma 2.4.10 in [25] shows that $\operatorname{coker}_{\mathcal{L}}(A)$ and $\mathcal{L} \otimes_{\mathcal{S}} \operatorname{coker}_{\mathcal{S}}(A)$ are isomorphic as $\mathcal{L}$-modules. Thus $\operatorname{dim}_{F} \operatorname{coker}_{\mathcal{L}}(A)$ does not exceed $\operatorname{dim}_{F} \operatorname{coker}_{\mathcal{S}}(A)$. 


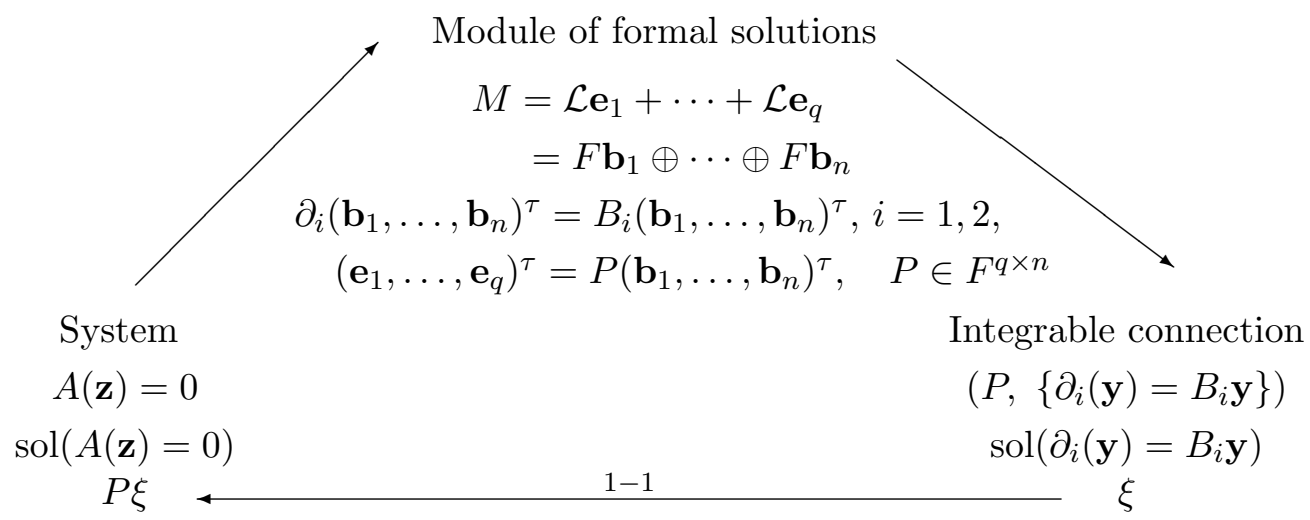

Fig. 1. Relationships among Systems, Modules and Solutions

Let $A(\mathbf{z})=0$ with $A \in \mathcal{S}^{p \times q}$ be a system of linear dimension $n$ and $M$ be its module of formal solutions with an $F$-basis $\mathbf{b}_{1}, \ldots, \mathbf{b}_{n}$. Suppose that $\partial_{i}\left(\mathbf{b}_{1}, \ldots, \mathbf{b}_{n}\right)^{\tau}=B_{i}\left(\mathbf{b}_{1}, \ldots, \mathbf{b}_{n}\right)^{\tau}$ where $B_{i} \in F^{n \times n}$ for $1 \leq i \leq m$. By a straightforward verification, $\left\{\partial_{i}(\mathbf{x})=B_{i} \mathbf{x}\right\}_{1 \leq i \leq m}$ is a fully integrable system, which is called the integrable connection of $A(\mathbf{z})=0$ with respect to the basis $\mathbf{b}_{1}, \ldots, \mathbf{b}_{n}$ of $M$.

$\partial$-finite and fully integrable systems are connected by the next proposition whose proof is given in [6, Proposition 2] and [25, Proposition 2.4.12].

Proposition 4.7 Let $A, \mathbf{b}_{1}, \ldots, \mathbf{b}_{n}, B_{1}, \ldots, B_{m}$ be as above, and $B$ be the stacking of the blocks $\left(\partial_{i} \cdot \mathbf{1}_{n}-B_{i}\right)$. Then

(i) $\operatorname{coker}_{\mathcal{L}}(A) \cong_{\mathcal{L}} \operatorname{coker}_{\mathcal{L}}(B)$.

(ii) Let $\left\{\mathbf{e}_{1}, \ldots, \mathbf{e}_{q}\right\}$ be the set of $\mathcal{L}$-generators of $M$ satisfying $A\left(\mathbf{e}_{1}, \ldots, \mathbf{e}_{q}\right)^{\tau}=0$ and $P \in F^{q \times n}$ be given by $\left(\mathbf{e}_{1}, \ldots, \mathbf{e}_{q}\right)^{\tau}=P\left(\mathbf{b}_{1}, \ldots, \mathbf{b}_{n}\right)^{\tau}$. Then, for any $\Delta$-extension $E$ of $F$, the correspondence $\xi \mapsto P \xi$ is an isomorphism of $C_{E}$-modules between $\operatorname{sol}_{E}\left(\left\{\partial_{i}(\mathbf{x})=B_{i} \mathbf{x}\right\}_{1 \leq i \leq m}\right)$ and $\operatorname{sol}_{E}(A(\mathbf{z})=0)$.

Remark that the inverse of the correspondence in Proposition 4.3 (ii) is given by $\eta \mapsto Q \eta$, where $Q$ is a matrix in $\mathcal{L}^{n \times q}$ such that $\left(\mathbf{b}_{1}, \ldots, \mathbf{b}_{n}\right)^{\tau}=Q\left(\mathbf{e}_{1}, \ldots, \mathbf{e}_{q}\right)^{\tau}$. From Proposition 4.7 (ii), all the solutions of the system $A(\mathbf{z})=0$ can be obtained from those of its integrable connection $\left\{\partial_{i}(\mathbf{x})=B_{i} \mathbf{x}\right\}_{1 \leq i \leq m}$, and vice versa. Figure 1 illustrates such a relationship, and it also suggests reducing the problem of solving $\partial$-finite systems to that of solving fully integrable systems.

\subsection{Fundamental matrices and Picard-Vessiot extensions}

Based on the discussion in Section 4.3., we generalize the notions and results of fundamental matrices and Picard-Vessiot extensions for $\partial$-finite systems.

Definition 4.8 Let $A(\mathbf{z})=0$ with $A \in \mathcal{S}^{p \times q}$ be a $\partial$-finite system, $M$ be its module of formal solutions, $\left\{\mathbf{e}_{1}, \ldots, \mathbf{e}_{q}\right\}$ be a set of $\mathcal{L}$-generators of $M$ and $\mathbf{b}_{1}, \ldots, \mathbf{b}_{n}$ be an $F$-basis of $M$ such that $A\left(\mathbf{e}_{1}, \ldots, \mathbf{e}_{q}\right)^{\tau}=0$ and $\left(\mathbf{e}_{1}, \ldots, \mathbf{e}_{q}\right)^{\tau}=P\left(\mathbf{b}_{1}, \ldots, \mathbf{b}_{n}\right)^{\tau}$ where $P \in F^{q \times n}$. 
$A q \times n$ matrix $V$ with entries in a $\Delta$-extension $E$ of $F$ is called a fundamental matrix for $A(\mathbf{z})=0$ if $V=P U$ where $U \in E^{n \times n}$ is a fundamental matrix of the integrable connection of $A(\mathbf{z})=0$ with respect to $\mathbf{b}_{1}, \ldots, \mathbf{b}_{n}$.

A Picard-Vessiot ring for an integrable connection of $A(\mathbf{z})=0$ is called a Picard-Vessiot ring for $A(\mathbf{z})=0$.

As a consequence of Theorem 3.5, we have

Theorem 4.9 Every $\partial$-finite system $A(\mathbf{z})=0$ over $F$ has a Picard-Vessiot ring $E$. If $F$ has characteristic 0 and $C_{F}$ is algebraically closed, then $C_{E}=C_{F}$.

Assume that $F$ has characteristic 0 with an algebraically closed field of constants. If $E$ is a Picard-Vessiot ring for the system $A(\mathbf{z})=0$ then the dimension of $\operatorname{sol}_{E}(A(\mathbf{z})=0)$ as a $C_{F}$-vector space equals the linear dimension of $A(\mathbf{z})=0$, whenever the latter is finite.

Example 4.10 Let $F, \delta_{x}, \sigma_{k}$ be as in Example 3.2, and $\mathcal{A}:=\left\{\delta_{x}(\mathbf{z})=A_{x} \mathbf{z}, \sigma_{k}(\mathbf{z})=A_{k} \mathbf{z}\right\}$ where

$$
\begin{gathered}
A_{x}=\left(\begin{array}{ccc}
\frac{x+1}{x} & \frac{k(x+1-k)}{x^{2}(k-1)} & -\frac{k(x+1-k)}{x^{2}(k-1)} \\
x+1 & \frac{x k-k^{2}+2 x^{2}+k x^{2}+k-1}{x(k-1)} & -\frac{x k-k^{2}+2 x^{2}+k x^{2}}{x(k-1)} \\
x+1 & \frac{x k+2 x^{2}+k x^{2}-2 k^{2}+k}{x(k-1)} & -\frac{x k+2 x^{2}+k x^{2}-2 k^{2}+1}{x(k-1)}
\end{array}\right), \\
A_{k}=\left(\begin{array}{ccc}
\frac{k+1}{k} & \frac{k+1-x k-x}{x(k-1)} & \frac{x k+x-k-1}{x(k-1)} \\
\frac{x(k+1)}{k} & \frac{1-2 x+k-x k+x^{3}}{k-1} & \frac{2 x+x k-x^{3}-k-1}{k-1} \\
\frac{x(k+1)}{k} & \frac{1-2 x k-2 x+k+x^{3}}{k-1} & \frac{2 x k+2 x-k-x^{3}-1}{k-1}
\end{array}\right),
\end{gathered}
$$

and $\mathbf{z}=\left(z_{1}, z_{2}, z_{3}\right)^{\tau}$. Note that $A_{k}$ is singular, so $\mathcal{A}$ is integrable but not fully integrable. We will show in Example 5.4 that all solutions of $\mathcal{A}$ can be found by a change of variable $\mathbf{z}=P \mathbf{y}$ where

$$
P=\left(\begin{array}{cc}
1 & 0 \\
0 & 1 \\
\frac{x(k-1)}{x^{2}-1} & \frac{x^{2}-k}{x^{2}-1}
\end{array}\right)
$$

and $\mathbf{y}$ is a solution of the fully integrable system $\mathcal{B}:\left\{\delta_{x}(\mathbf{y})=B_{x} \mathbf{y}, \sigma_{k}(\mathbf{y})=B_{k} \mathbf{y}\right\}$ with

$$
B_{x}=\left(\begin{array}{cc}
\frac{-x+x^{3}-1+x^{2}-x k-k+k^{2}}{x\left(x^{2}-1\right)} & \frac{k(x+1-k)}{x^{2}\left(x^{2}-1\right)} \\
\frac{-x-x k+x^{3}-1-x^{2}+k^{2}-k x^{2}}{x^{2}-1} & \frac{-k^{2}+x k+k x^{2}+3 x^{2}-1}{x\left(x^{2}-1\right)}
\end{array}\right)
$$

and

$$
B_{k}=\left(\begin{array}{cc}
\frac{x k+x+k^{2}+2 k+1}{k(x+1)} & -\frac{k+1}{x(x+1)} \\
-\frac{\left(k x^{2}-x-k^{2}-2 k-1\right) x}{k(x+1)} & \frac{x^{2}+x-1-k}{x+1}
\end{array}\right) .
$$

So it suffices to compute a Picard-Vessiot extension of $\mathcal{B}$. The same method to construct a fundamental matrix for the system in Example 3.2 yields a fundamental matrix for $\mathcal{B}$ :

$$
U=\left(\begin{array}{cc}
x k e^{x} & -k x^{k} \\
k x^{2} e^{x} & \left(x^{2}-k-1\right) x^{k+1}
\end{array}\right),
$$

hence $P U$ is for $\mathcal{A}$. In addition, a Picard-Vessiot ring $\mathbb{C}(x, k)\left[e^{x}, e^{-x}, x^{k}, x^{-k}\right]$ for $\mathcal{B}$ is a Picard-Vessiot ring for $\mathcal{A}$. 


\section{Computing linear dimension}

We now describe how to compute linear dimension for a given linear functional system $A(\mathbf{z})=0$.

Let $N$ be a submodule of a free $\mathcal{L}$-module $\mathcal{L}^{q}$ with a finite set of generators. One can compute a Gröbner basis of $N$ over $\mathcal{L}$ (see [?] and [25, Chapter 3]), which gives rise to an $F$ basis of $\mathcal{L}^{q} / N$. Thus, one can determine whether a linear functional system is $\partial$-finite, and construct an $F$-basis of its module of formal solutions.

The following proposition indicates that the same goal may be achieved by Gröbner basis computation over Ore algebra $\mathcal{S}$ (see [8]) if $\operatorname{coker}_{\mathcal{S}}(A)$ has finite dimension over $F$. Notice that the linear dimension of $A(\mathbf{z})=0$ never exceeds $\operatorname{dim}_{F} \operatorname{coker}_{\mathcal{S}}(A)$ by Remark 4.6.

Proposition 5.1 Let $N$ be a left submodule of $\mathcal{S}^{1 \times n}$ such that $\mathcal{S}^{1 \times n} / N$ has finite dimension over $F$ and $\bar{N}$ the submodule generated by $N$ in $\mathcal{L}^{1 \times n}$. Then $\mathcal{S}^{1 \times n} /\left(\bar{N} \cap \mathcal{S}^{1 \times n}\right)$ and $\mathcal{L}^{1 \times n} / \bar{N}$ are isomorphic as F-vector spaces.

The proof to Proposition 5.1 can be found in [25, Proposition 2.4.6].

Another useful fact is described in the next proposition whose proof is found in $[25$, Proposition 2.4.11(ii)].

Proposition 5.2 Suppose that $\operatorname{coker}_{\mathcal{S}}(A)$ with $A \in \mathcal{S}^{p \times q}$ has a finite $F$-basis $\mathbf{f}_{1}, \ldots, \mathbf{f}_{d}$ and $\partial_{i}\left(\mathbf{f}_{1}, \ldots, \mathbf{f}_{d}\right)^{\tau}=D_{i}\left(\mathbf{f}_{1}, \ldots, \mathbf{f}_{d}\right)^{\tau}$ where $D_{i} \in F^{d \times d}$. Let $D$ be the stacking of the blocks $\left(\partial_{i} \cdot \mathbf{1}_{d}-D_{i}\right)$. Then $\operatorname{coker}_{\mathcal{S}}(A) \cong_{\mathcal{S}} \operatorname{coker}_{\mathcal{S}}(D)$ and $\operatorname{coker}_{\mathcal{L}}(A) \cong_{\mathcal{L}} \operatorname{coker}_{\mathcal{L}}(D)$.

The above proposition reveals that, to compute linear dimension of a system $A(\mathbf{z})=0$ such that $\operatorname{coker}_{\mathcal{S}}(A)$ has finite dimension over $F$, it suffices to compute linear dimension of the (integrable) system $\left\{\partial_{i}(\mathbf{y})=D_{i} \mathbf{y}\right\}_{1 \leq i \leq m}$ in which $\mathbf{y}=\left(y_{1}, \ldots, y_{d}\right)^{\tau}$.

In the situation described in Proposition 5.2, let $H_{0}$ be the set of row vectors of $D, N_{0}$ the submodule generated by $H_{0}$ over $\mathcal{S}$, and $N$ the submodule generated by $H_{0}$ over $\mathcal{L}$. We proceed as follows to compute a Gröbner basis of $N$ over $\mathcal{L}$, which gives rise to an $F$-basis of $\mathcal{L}^{d} / N$, the module of formal solutions of $\left\{\partial_{i}(\mathbf{y})=D_{i} \mathbf{y}\right\}_{1 \leq i \leq m}$.

Compute the rank of $D_{j}$ for each $j$ with $\ell+1 \leq j \leq m$. If each $D_{j}$ has full rank, then $\left\{\partial_{i}(\mathbf{y})=D_{i} \mathbf{y}\right\}_{1 \leq i \leq m}$ is fully integrable and so $H_{0}$ is already a Gröbner basis of $N$ over $\mathcal{L}$ (see [25, Example 3.2.4]). Otherwise, the row vectors of some $D_{j}$ satisfy certain linear relations over $F$, which, together with $\partial_{j}(\mathbf{y})=D_{j} \mathbf{y}$, implies $F$-linear relations among $\partial_{j}\left(y_{1}\right), \ldots \partial_{j}\left(y_{d}\right)$. Assume that one of the relations is

$$
f_{1} \partial_{j}\left(y_{1}\right)+\cdots+f_{d} \partial_{j}\left(y_{d}\right)=0
$$

where $j \in\{\ell+1, \ldots, m\}$ and $f_{1}, \ldots f_{d} \in F$, not all zero. Applying $\partial_{j}^{-1}$ to it yields

$$
\sigma_{j}^{-1}\left(f_{1}\right) y_{1}+\cdots+\sigma_{j}^{-1}\left(f_{d}\right) y_{d}=0 .
$$

Hence the vector $\left(\sigma_{j}^{-1}\left(f_{1}\right), \ldots, \sigma_{j}^{-1}\left(f_{d}\right)\right)$ belongs to the $\mathcal{L}$-submodule $N$, but it does not belong to $N_{0}$. Adding to $H_{0}$ the new vectors obtained from the linear relations of the form (4), we have a new set $H_{1}$ of generators for $N$. Now we compute a Gröbner basis of $N$ over $\mathcal{L}$ using $H_{1}$. By Lemmas 2.5.1 and 2.5.2 in [25], such a basis can be computed by merely rank computation, Gaussian elimination and the "Reduce All" trick. It is unnecessary to form 
any $S$-polynomials. This simplification is due to the integrability of $\left\{\partial_{i}(\mathbf{y})=D_{i} \mathbf{y}\right\}_{1 \leq i \leq m}$. A detailed description of this process is formulated as an algorithm named LinearReduction in $[25, \S 2.5]$.

We now give some examples to compute linear dimensions.

Example 5.3 Let $A_{1}, \ldots, A_{m}$ be in $F^{n \times n}$ and

$$
A=\left(\begin{array}{c}
\partial_{1} \cdot \mathbf{1}_{n}-A_{1} \\
\vdots \\
\partial_{m} \cdot \mathbf{1}_{n}-A_{m}
\end{array}\right) \in \mathcal{S}^{m n \times n} .
$$

The system $A(\mathbf{z})=0$ corresponds to the system $\left\{\partial_{i}(\mathbf{z})=A_{i} \mathbf{z}\right\}_{1 \leq i \leq m}$. Let $M$ be the module of formal solutions of $A(\mathbf{z})=0$, and $\mathbf{e}_{1}, \ldots, \mathbf{e}_{n}$ be the respective images of $\mathbf{e}_{1 n}, \ldots, \mathbf{e}_{n n}$ in $M$. For $\mathbf{e}:=\left(\mathbf{e}_{1}, \ldots, \mathbf{e}_{n}\right)^{\tau} \in M^{n}$, we have $A(\mathbf{e})=0$ or $\partial_{i}(\mathbf{e})=A_{i} \mathbf{e}$ for each $i$. Since the entries of $A_{i}$ are in $F, \partial_{i}\left(\mathbf{e}_{j}\right) \in \sum_{s=1}^{n} F \mathbf{e}_{s}$ for all $i, j$, thus $\mathcal{L} \mathbf{e}_{j} \subseteq \sum_{s=1}^{n} F \mathbf{e}_{s}$ for all $j$. So $M=\sum_{s=1}^{n} \mathcal{L} \mathbf{e}_{s}=\sum_{s=1}^{n} F \mathbf{e}_{s}$. In particular, $\operatorname{dim}_{F} M \leq n$. Observe that $\operatorname{dim}_{F} M=n$ if and only if the system $\left\{\partial_{i}(\mathbf{z})=A_{i} \mathbf{z}\right\}_{1 \leq i \leq m}$ is fully integrable (see [25, Proposition 2.4.9]).

Example 5.4 Let $F, \delta_{x}, \sigma_{k}$ and the system $\mathcal{A}$ be given in Example 4.10. We follow the idea of the algorithm LinearReduction to compute linear dimension of $\mathcal{A}$. Note that $A_{k}$ is singular. Solve the linear system $\left(v_{1}, v_{2}, v_{3}\right) A_{k}=0$ in $v_{1}, v_{2}, v_{3}$. A nontrivial solution of this system yields $\sigma_{k}\left(z_{3}\right)=\frac{x k}{x^{2}-1} \sigma_{k}\left(z_{1}\right)+\frac{x^{2}-k-1}{x^{2}-1} \sigma_{k}\left(z_{2}\right)$. By an application of $\sigma_{k}^{-1}$, we get $z_{3}=\frac{x(k-1)}{x^{2}-1} z_{1}+\frac{x^{2}-k}{x^{2}-1} z_{2}$, thus,

$$
\left(\begin{array}{c}
z_{1} \\
z_{2} \\
z_{3}
\end{array}\right)=\underbrace{\left(\begin{array}{cc}
1 & 0 \\
0 & 1 \\
\frac{x(k-1)}{x^{2}-1} & \frac{x^{2}-k}{x^{2}-1}
\end{array}\right)}_{P}\left(\begin{array}{c}
z_{1} \\
z_{2}
\end{array}\right) .
$$

Substitute this relation into $\mathcal{A}$, we get $\delta_{x}\left(z_{1}, z_{2}\right)^{\tau}=B_{x}\left(z_{1}, z_{2}\right)^{\tau}$ and $\sigma_{k}\left(z_{1}, z_{2}\right)^{\tau}=B_{k}\left(z_{1}, z_{2}\right)^{\tau}$ where

$$
B_{x}=\left(\begin{array}{cc}
\frac{-x+x^{3}-1+x^{2}-x k-k+k^{2}}{x} & \frac{k(x+1-k)}{x^{2}\left(x^{2}-1\right)} \\
\frac{-x-x k+x^{3}-1-x^{2}+k^{2}-k x^{2}}{x^{2}-1} & \frac{-k^{2}+x k+k x^{2}+3 x^{2}-1}{x\left(x^{2}-1\right)}
\end{array}\right) \text { and } B_{k}=\left(\begin{array}{cc}
\frac{x k+x+k^{2}+2 k+1}{k(x+1)} & -\frac{k+1}{x(x+1)} \\
-\frac{\left(k x^{2}-x-k^{2}-2 k-1\right) x}{k(x+1)} & \frac{x^{2}+x-1-k}{x+1}
\end{array}\right) \text {. }
$$

A straightforward calculation verifies that the first-order system $\mathcal{B}$ given by $B_{x}$ and $B_{k}$ is fully integrable, so $\mathcal{B}$ has linear dimension two by Example 5.3. According to the algorithm LinearReduction, all the solutions $\mathbf{z}$ of $\mathcal{A}$ can be obtained from the solutions $\mathbf{y}$ of $\mathcal{B}$ via a change of variable $\mathbf{z}=P \mathbf{y}$, and the modules of formal solutions for $\mathcal{A}$ and for $\mathcal{B}$ are the same. Hence $\mathcal{A}$ has linear dimension two.

The following example shows that there are $\partial$-finite systems whose $\mathcal{S}$-cokernels are infinite-dimensional over $F$.

Example 5.5 Let $A=\left(L_{1}, L_{2}\right)^{\tau}$ with $L_{1}=\partial_{1} \partial_{2}\left(\partial_{1}+1\right)$ and $L_{2}=\partial_{1} \partial_{2}\left(\partial_{2}+1\right)$, $J$ the ideal in $\mathcal{S}$ generated by $L_{1}$ and $L_{2}$, and $M=\operatorname{coker}_{\mathcal{L}}(A)$. Since $\partial_{1}$ and $\partial_{2}$ are invertible in $\mathcal{L}$, then $M=\mathcal{L} /\left(\mathcal{L}\left(\partial_{1}+1\right)+\mathcal{L}\left(\partial_{2}+1\right)\right)$, thus $\operatorname{dim}_{F} M=1$. However, $\mathcal{S} / J$ is infinite-dimensional over $F$. 
We now conclude how to determine whether a linear functional system is $\partial$-finite. As seen in Examples 5.3 and 5.4, when the system is given as an integrable system, we have a set of generators of $M$ over $F$, so computing $\operatorname{dim}_{F} M$ can be done by linear algebra. In particular, when $A(\mathbf{z})=0$ is given by a finite-rank ideal in $\mathcal{S}$, Proposition 5.1 shows that either $M=0$ (if the ideal contains a monomial in $\partial_{\ell+1}, \ldots, \partial_{m}$ ) or an $F$-basis of $M$ can be computed via Gröbner bases of ideals in $\mathcal{S}$. There are algorithms and implementations for this task $[7,8]$. For a more general matrix $A \in \mathcal{S}^{p \times q}$, one can use the Gröbner basis technique developed in [25, Chapter 3] for computing $F$-bases of $\mathcal{L}$-modules. However, to compute the linear dimension of $A(\mathbf{z})=0$ for which $\operatorname{coker}_{\mathcal{S}}(A)$ is finite-dimensional it suffices to compute the linear dimension of an integrable system according to Proposition 5.2. The algorithm LinearReduction supplies a tool for the latter task. Therefore, Gröbner basis techniques in $\mathcal{L}$ are necessary only when $\operatorname{coker}_{\mathcal{S}}(A)$ is infinite-dimensional over $F$.

\section{Factorization of Laurent-Ore modules}

The work of this section is motivated by the algorithm FactorWithSpecifiedLeaders in $[13,14]$, where the idea of associated equations is extended to factor linear partial differential equations with finite-dimensional solution spaces. In terms of modules over an Ore algebra $\mathcal{S}=F\left[\partial_{1}, \ldots, \partial_{m}\right]$ where $\ell=m$, the problem solved by their algorithm can be formulated as follows: given a submodule $N$ of $\mathcal{S}^{n}$ such that $M=\mathcal{S}^{n} / N$ is finite-dimensional over the field $F$, finds all submodules of $\mathcal{S}^{n}$ that contain $N$. Such a submodule is called a factor of $N$ since all its solutions are solutions of $N$. In their algorithm a factor is represented by a Gröbner basis with respect to a pre-chosen monomial order. Observe that, for a (right) factor of a given order, there is only one possibility for its leading derivative in the ordinary case, whereas, there are many possibilities in the partial case. Due to this complication, the algorithm has to check every possibility to compute all the factors of a given order. In this ideal-theoretic approach the quotient module $M$ does not come into play.

In the module-theoretic approach to be described in this section, we compute all submodules of the above quotient module $M$, and then recover the factors of $N$ in the sense of $[13,14]$ via the canonical map from $\mathcal{S}^{n}$ to $M$. As all submodules of $M$ are represented by linear bases over $F$, the problem of guessing leading derivatives goes away. The same idea carries over to Laurent-Ore modules and results in a factorization algorithm for $\partial$-finite systems.

\subsection{Constructions with modules over Laurent-Ore algebras}

Given a ring $R$, we first review some notions of reducibility of $R$-modules defined in [22].

An $R$-module $M$ is reducible if $M$ has a submodule other than 0 and $M$. Otherwise, $M$ is irreducible or simple. An $R$-module $M$ is completely reducible or semisimple if for every submodule $N_{1}$ there exists a submodule $N_{2}$ such that $M=N_{1} \oplus N_{2}$. Note that an irreducible module is completely reducible as well. An $R$-module $M$ is decomposable if $M$ can be written as $N_{1} \oplus N_{2}$ where $N_{1}$ and $N_{2}$ are nontrivial submodules of $M$. Otherwise, $M$ is indecomposable. Clearly, an $R$-module $M$ is reducible if it is decomposable, and $M$ is irreducible when it is both indecomposable and completely reducible. By factoring an $R$ module, we mean finding its $R$-submodules.

As before, let $F$ be a $\Delta$-field with $C$ the field of constants, $\mathcal{S}=F\left[\partial_{1}, \ldots, \partial_{m}\right]$ and 
$\mathcal{L}=F\left[\partial_{1}, \ldots, \partial_{m}, \partial_{\ell+1}^{-1}, \ldots, \partial_{m}^{-1}\right]$ be the corresponding Ore algebra and Laurent-Ore algebra, respectively. In the sequel, unless otherwise specified, $F$ has characteristic 0 and $C$ is algebraically closed.

Clearly, ordinary and partial differential modules in [20] are special cases of $\mathcal{L}$-modules. The constructions in $[20, \S 2.2]$ can be carried on $\mathcal{L}$-modules in a similar way.

Let $M$ be an $\mathcal{L}$-module and $N$ a submodule of $M$. The $F$-vector space $M / N$ with the induced actions: $\partial_{i}(\mathbf{w}+N)=\partial_{i}(\mathbf{w})+N$ for $1 \leq i \leq m$ and $\partial_{j}^{-1}(\mathbf{w}+N)=\partial_{j}^{-1}(\mathbf{w})+N$ for $\ell+1 \leq j \leq m$, is the quotient module.

The direct sum of two $\mathcal{L}$-modules $M_{1}$ and $M_{2}$ is $M_{1} \oplus M_{2}$ equipped with the actions: $\partial_{i}\left(\mathbf{w}_{1}+\mathbf{w}_{2}\right)=\partial_{i}\left(\mathbf{w}_{1}\right)+\partial_{i}\left(\mathbf{w}_{2}\right)$ and $\partial_{j}^{-1}\left(\mathbf{w}_{1}+\mathbf{w}_{2}\right)=\partial_{j}^{-1}\left(\mathbf{w}_{1}\right)+\partial_{j}^{-1}\left(\mathbf{w}_{2}\right)$ for $1 \leq i \leq m$ and $\ell+1 \leq j \leq m$.

The tensor product $M_{1} \otimes M_{2}$ of two $\mathcal{L}$-modules $M_{1}$ and $M_{2}$ is $M_{1} \otimes_{F} M_{2}$ equipped with the actions: $\partial_{i}\left(\mathbf{w}_{1} \otimes \mathbf{w}_{2}\right)=\partial_{i}\left(\mathbf{w}_{1}\right) \otimes \mathbf{w}_{2}+\mathbf{w}_{1} \otimes \partial_{i}\left(\mathbf{w}_{2}\right)$ for $i \leq \ell$, and $\partial_{j}^{\nu}\left(\mathbf{w}_{1} \otimes \mathbf{w}_{2}\right)=$ $\partial_{j}^{\nu}\left(\mathbf{w}_{1}\right) \otimes \partial_{j}^{\nu}\left(\mathbf{w}_{2}\right)$ for $j>\ell$ and $\nu \in\{-1,1\}$.

The $d$-th exterior power $\wedge^{d} M$ of an $\mathcal{L}$-module $M$ is the $F$-vector space $\wedge_{F}^{d} M$ provided with the actions given by the formulas $\partial_{i}\left(\mathbf{w}_{1} \wedge \cdots \wedge \mathbf{w}_{d}\right)=\sum_{s=1}^{d} \mathbf{w}_{1} \wedge \cdots \wedge\left(\partial_{i} \mathbf{w}_{s}\right) \wedge \cdots \wedge \mathbf{w}_{d}$ for $i \leq \ell$ and $\partial_{j}^{\nu}\left(\mathbf{w}_{1} \wedge \cdots \wedge \mathbf{w}_{d}\right)=\partial_{j}^{\nu}\left(\mathbf{w}_{1}\right) \wedge \cdots \wedge \partial_{j}^{\nu}\left(\mathbf{w}_{d}\right)$ for $j>\ell$ and $\nu \in\{-1,1\}$.

Exterior powers of Laurent-Ore modules play an important role in the next section.

\subsection{A module-theoretic approach to factorization}

We now describe an idea on factoring Laurent-Ore modules.

Recall that a decomposable ([17]) element $\mathbf{w} \in \wedge^{d} M$ is an exterior product of $d$ elements in $M$, i.e., $\mathbf{w}=\mathbf{w}_{1} \wedge \cdots \wedge \mathbf{w}_{d}$.

The following theorem generalizes Lemma 10 in [9] or the corresponding statement in [20, page 111]:

Theorem 6.1 A Laurent-Ore module $M$ has a d-dimensional submodule if and only if $\wedge^{d} M$ has a one-dimensional submodule generated by a decomposable element.

Remark that the operators $\partial_{j}^{-1}$ are indispensable in the proof of Theorem 6.1 (see also [25, Theorem 4.3.1]), and this proof yields a correspondence between $d$-dimensional submodules and one-dimensional submodules generated by decomposable elements: if a $d$-dimensional submodule of $M$ has an $F$-basis $\mathbf{v}_{1}, \ldots, \mathbf{v}_{d}$, then the linear subspace generated by $\mathbf{v}_{1} \wedge \cdots \wedge \mathbf{v}_{d}$ in $\wedge^{d} M$ is a one-dimensional submodule; conversely, if a one-dimensional submodule of $\wedge^{d} M$ is generated by a decomposable element $\mathbf{v}_{1} \wedge \cdots \wedge \mathbf{v}_{d}$, then the $F$-linear subspace generated by $\mathbf{v}_{1}, \ldots, \mathbf{v}_{d}$ in $M$ is a $d$-dimensional submodule.

Let $M$ be a Laurent-Ore module with an $F$-basis $\left\{\mathbf{e}_{1}, \ldots, \mathbf{e}_{n}\right\}$. Set $g=\left(\begin{array}{l}n \\ d\end{array}\right)$. Then the module $\wedge^{d} M$ has an $F$-basis $\left\{\mathbf{f}_{1}, \ldots, \mathbf{f}_{g}\right\}$. Let $\mathbf{e}=\left(\mathbf{e}_{1}, \ldots, \mathbf{e}_{n}\right)$ and $\mathbf{f}=\left(\mathbf{f}_{1}, \ldots, \mathbf{f}_{g}\right)$. By Theorem 6.1, the problem of finding $d$-dimensional submodules of $M$ is converted into that of finding one-dimensional submodules of $\wedge^{d} M$ whose generators are decomposable, and thus the factorization problem is reduced to two "subproblems": finding one-dimensional submodules and deciding the decomposability of their generators.

The first subproblem can be solved by a recursive method [15] for determining onedimensional submodules of a Laurent-Ore module. Applying the method to $\wedge^{d} M$ yields several finite subsets $S_{1}, \ldots, S_{t} \subset F^{g}$ with the following properties:

(a) The elements of each $S_{k}$ are $C$-linearly independent. 
(b) A one-dimensional $F$-space of $\wedge^{d} M$ is a submodule if and only if it is an $F$-space generated by the product of $\mathbf{f}$ and a nontrivial $C$-linear combination of elements of some $S_{k}$.

Now we deal with the second subproblem. Let $S$ be one of the $S_{k}$ with $q$ elements, and w be the product of $\mathbf{f}$ and a $C$-linear combination of elements of $S$, in which the coefficients are unspecified constants $c_{1}, \ldots, c_{q}$. Consider the map $\phi_{\mathbf{w}}: M \rightarrow \wedge^{d+1} M$ given by $\mathbf{v} \mapsto \mathbf{v} \wedge \mathbf{w}$. By Theorem 1.1 in [17, Chapter 4] and the proof of Theorem 6.1, $\mathbf{w}$ is decomposable if and only if $\operatorname{ker}\left(\phi_{\mathbf{w}}\right)$ is of dimension $d$. The latter is equivalent to the condition that the matrix $P$ of $\phi_{\mathbf{w}}$ has rank $(n-d)$. Hence, testing the decomposability of $\mathbf{w}$ amounts to a rank computation of $P$, i.e., identifying the unspecified constants $c_{1}, \ldots, c_{q}$ such that the rank of $P$ is $(n-d)$. This further amounts to solving several systems consisting of homogeneous polynomial equations and inequations in $c_{1}, \ldots, c_{q}$ over $F$. Using a linear basis of $F$ over $C$, we can translate every such system into finitely many subsystems over $C$. Each subsystem has two portions: a set of polynomial equations and an inequation. If none of the subsystems has a solution, then the product of $\mathbf{f}$ and any $C$-linear combination of elements of $S$ is not decomposable and thus does not lead to any $d$-dimensional submodule of $M$. Otherwise, substitute a solution into the matrix $P$, and compute a basis $\mathbf{r}_{1}, \ldots, \mathbf{r}_{d}$ of the rational kernel of $P$ where $\mathbf{r}_{j} \in F^{n}$. Set $\mathbf{u}_{j}=\mathbf{e} \mathbf{r}_{j}$ for $j=1, \ldots, d$. Then $\oplus_{j=1}^{d} F \mathbf{u}_{j}$ is a $d$-dimensional submodule of $M$.

A few words need to be said about those subsystems derived from the rank condition for $P$, since they may have infinitely many solutions after dehomogenization. We require that the substitution of any solution of a subsystem into $P$ not only yields the required rank for $P$, but also makes a fixed $(n-d) \times(n-d)$ minor nonzero. An $(n-d) \times(n-d)$ minor may correspond to several subsystems. This requirement can always be fulfilled, and will help us describe all $d$-submodules of $M$ by a finite amount of information. We proceed as follows. Let $T$ be such a subsystem. Using the nonzero minor corresponding to $T$ and Cramer's rule, we may find a basis $\mathbf{r}_{1}, \ldots, \mathbf{r}_{d}$ of the rational kernel of $P$ where the entries of the $\mathbf{r}_{j}$ are in $F\left(c_{1}, \ldots, c_{q}\right)$ and their denominators divide the given minor. Set $\mathbf{u}_{j}=\mathbf{e r}_{j}$ for $j=1, \ldots, d$. Then $\oplus_{j=1}^{d} F \mathbf{u}_{j}$ represents all $d$-dimensional submodules obtained by substituting solutions of $T$ for $c_{1}, \ldots, c_{q}$ into $\mathbf{u}_{1}, \ldots, \mathbf{u}_{d}$. Note that we may check the set of solutions of $T$ by techniques from computational algebraic geometry. These considerations lead to a method for computing all submodules of $M$, which is described stepwise in [25, §4.4].

Remark 6.2 The above representation for d-dimensional submodules of $M$ is rather naive and has a lot of redundancy. To have more concise representations, one would partition $d$ dimensional submodules with respect to module isomorphism, and generalize the techniques given in [20, page 112].

We now present two examples for factoring Laurent-Ore modules.

Example 6.3 [Legendre's system] Let $F, \delta_{x}, \sigma_{k}$ be as given in Example 3.2 and $\mathcal{L}=$ $F\left[\partial_{x}, \partial_{k}, \partial_{k}^{-1}\right]$ be the Laurent-Ore algebra. A Gröbner basis of the ideal generated by the Legendre's system (1) is

$$
g_{1}=x k+x+\left(x^{2}-1\right) \partial_{x}-(k+1) \partial_{k} \quad \text { and } \quad g_{2}=k+1+(k+2) \partial_{k}^{2}-(2 x k+3 x) \partial_{k} .
$$


Let $A=\left(g_{1}, g_{2}\right)^{\tau} \in \mathcal{L}^{2 \times 1}, M=\mathcal{L} /\left(\mathcal{L} g_{1}+\mathcal{L} g_{2}\right)$ and $\mathbf{e}_{1}, \mathbf{e}_{2}$ be the images of 1 and $\partial_{k}$ in $M$, respectively. Then $\mathbf{e}_{1}, \mathbf{e}_{2}$ form a basis of $M$ over $F$ and, in addition,

$$
\partial_{x}\left(\begin{array}{l}
\mathbf{e}_{1} \\
\mathbf{e}_{2}
\end{array}\right)=\left(\begin{array}{cc}
\frac{-x k-x}{x^{2}-1} & \frac{k+1}{x^{2}-1} \\
\frac{-k-1}{x^{2}-1} & \frac{x k+x}{x^{2}-1}
\end{array}\right)\left(\begin{array}{l}
\mathbf{e}_{1} \\
\mathbf{e}_{2}
\end{array}\right), \quad \partial_{k}\left(\begin{array}{l}
\mathbf{e}_{1} \\
\mathbf{e}_{2}
\end{array}\right)=\left(\begin{array}{cc}
0 & 1 \\
\frac{-k-1}{k+2} & \frac{2 x k+3 x}{k+2}
\end{array}\right)\left(\begin{array}{l}
\mathbf{e}_{1} \\
\mathbf{e}_{2}
\end{array}\right) .
$$

Apply the algorithm in [15], we find that $M$ has no one-dimensional submodules, so $M$ is irreducible.

Example 6.4 Let $F, \delta_{x}, \sigma_{k}$ be as given in Example 3.2 and $\mathcal{L}=F\left[\partial_{x}, \partial_{k}, \partial_{k}^{-1}\right]$ the LaurentOre algebra. Let $M$ be an $\mathcal{L}$-module with an $F$-basis $\left\{\mathbf{e}_{1}, \mathbf{e}_{2}, \mathbf{e}_{3}, \mathbf{e}_{4}\right\}$ satisfying $\partial_{x}\left(\mathbf{e}_{1}, \mathbf{e}_{2}, \mathbf{e}_{3}, \mathbf{e}_{4}\right)^{\tau}$ $=A_{x}\left(\mathbf{e}_{1}, \mathbf{e}_{2}, \mathbf{e}_{3}, \mathbf{e}_{4}\right)^{\tau}$ and $\partial_{k}\left(\mathbf{e}_{1}, \mathbf{e}_{2}, \mathbf{e}_{3}, \mathbf{e}_{4}\right)^{\tau}=A_{k}\left(\mathbf{e}_{1}, \mathbf{e}_{2}, \mathbf{e}_{3}, \mathbf{e}_{4}\right)^{\tau}$ where

$$
A_{x}=\left(\begin{array}{cccc}
0 & 1 & 0 & 0 \\
\frac{-x^{3}-x^{2} k+2 x^{2}+x k+k^{2} x+k^{2}+k^{3}}{x^{2}(-x+k)} & \frac{2\left(x^{2}-x-k^{2}\right)}{(x-k) x} & 0 & 0 \\
0 & 0 & 0 & 1 \\
0 & 0 & \frac{-x^{3}-x^{2} k+x^{2}+3 x k+2 x+k^{2} x+4 k^{2}+5 k+2+k^{3}}{x^{2}(-x+k+1)} & -\frac{2\left(-x^{2}+x+k^{2}+2 k+1\right)}{(-x+k+1) x}
\end{array}\right)
$$

and

$$
A_{k}=\left(\begin{array}{cccc}
0 & 0 & 1 & 0 \\
0 & 0 & 0 & 1 \\
-\frac{(x-k) x^{2}}{x-k-2} & 0 & \frac{2 x(x-k-1)}{x-k-2} & 0 \\
-\frac{2 x\left(x^{2}-2 x k-3 x+k^{2}+2 k\right)}{(x-k-2)^{2}} & -\frac{(x-k) x^{2}}{x-k-2} & \frac{2\left(x^{2}-2 x k-4 x+k^{2}+3 k+2\right)}{(x-k-2)^{2}} & \frac{2 x(x-k-1)}{x-k-2}
\end{array}\right) .
$$

Let us compute all two-dimensional submodules of $M$. Clearly,

$$
\mathbf{f}_{1}=\mathbf{e}_{1} \wedge \mathbf{e}_{2}, \mathbf{f}_{2}=\mathbf{e}_{1} \wedge \mathbf{e}_{3}, \mathbf{f}_{3}=\mathbf{e}_{1} \wedge \mathbf{e}_{4}, \mathbf{f}_{4}=\mathbf{e}_{2} \wedge \mathbf{e}_{3}, \mathbf{f}_{5}=\mathbf{e}_{2} \wedge \mathbf{e}_{4}, \mathbf{f}_{6}=\mathbf{e}_{3} \wedge \mathbf{e}_{4}
$$

form a basis of $\wedge^{2} M$ over $F$. By the algorithm in [15], every one-dimensional submodule of $\wedge^{2} M$ has a generator of the form $\mathbf{w}=\left(\mathbf{f}_{1}, \mathbf{f}_{2}, \mathbf{f}_{3}, \mathbf{f}_{4}, \mathbf{f}_{5}, \mathbf{f}_{6}\right)\left(\sum_{i=1}^{6} c_{i} \mathbf{v}_{i}\right)$ where

$$
\mathbf{v}_{1}=\left(\begin{array}{c}
-x^{2}+2 x k-k^{2} \\
-\frac{2 k^{3}+k^{2}-2 x^{2}-x^{3}+x+k^{4}-2 x^{2} k^{2}+3 x k+3 k^{2} x-4 x^{2} k+x^{4}}{x^{3}} \\
\frac{x+2 x^{3}+k^{3}+2 x k+k^{2}-3 x^{2} k-3 x^{2}}{x^{2}} \\
\frac{-2 x k+k+k^{2} k+2 k^{2}-x-2 k^{2} x}{x^{2}} \\
-\frac{k+k^{2}+x^{2}-x-2 x k}{x} \\
-\frac{x^{2}-2 x k-2 x+k^{2}+2 k+1}{x^{2}}
\end{array}\right),
$$

and the other expressions $\mathbf{v}_{2}, \ldots, \mathbf{v}_{6}$ are quite big and given in [25, pages 70-71].

It remains to determine the decomposability of $\mathbf{w}$. Consider the map $M \rightarrow \wedge^{3} M$ given by $\mathbf{v} \mapsto \mathbf{v} \wedge \mathbf{w}$, whose matrix is some $P \in F^{4 \times 4}$ (we do not write down this matrix explicitly due to its big size). The matrix $P$ has rank 2 if and only if all its $3 \times 3$ minors are zero and 
there exists a nonzero $2 \times 2$ minor. This yields four sets of solutions for the $c_{i}$ :

$$
\begin{gathered}
\left\{c_{1}=c_{1}, \quad c_{2}=c_{2}, \quad c_{3}=0, \quad c_{4}=c_{4}, \quad c_{5}=0, \quad c_{6}=0\right\}, \\
\left\{c_{1}=c_{1}, \quad c_{2}=c_{2}, \quad c_{3}=c_{3}, \quad c_{4}=-\frac{3 c_{3}}{2}, \quad c_{5}=0, \quad c_{6}=0\right\}, \\
\left\{c_{1}=\frac{c_{3}\left(2 c_{4}+3 c_{3}-4 c_{5}\right)}{4 c_{5}}, \quad c_{2}=c_{2}, \quad c_{3}=c_{3}, \quad c_{4}=c_{4}, \quad c_{5}=c_{5}, \quad c_{6}=0\right\}, \\
\left\{c_{1}=c_{1}, c_{2}=-\frac{8 c_{6} c_{5}-2 c_{3} c_{4}-3 c_{3}^{2}-4 c_{6} c_{4}-20 c_{3} c_{6}-4 c_{1} c_{6}+4 c_{1} c_{5}+4 c_{3} c_{5}-24 c_{6}^{2}}{4 c_{6}}, c_{3}=c_{3}, c_{4}=c_{4}, c_{5}=c_{5}, c_{6}=c_{6}\right\} .
\end{gathered}
$$

Therefore $M$ has two-dimensional submodules if and only if the $c_{i}$ in $\mathbf{w}$ satisfy one of these four relations.

Substitute these four relations into $P$ respectively and compute the corresponding $F$-bases for the rational kernel of $P$. Finally, we get all two-dimensional submodules:

$$
N_{i}=\left\{a_{1} \mathbf{u}_{i, 1}+a_{2} \mathbf{u}_{i, 2} \mid a_{1}, a_{2} \in F\right\}, \quad i=1,2,3,4,
$$

where

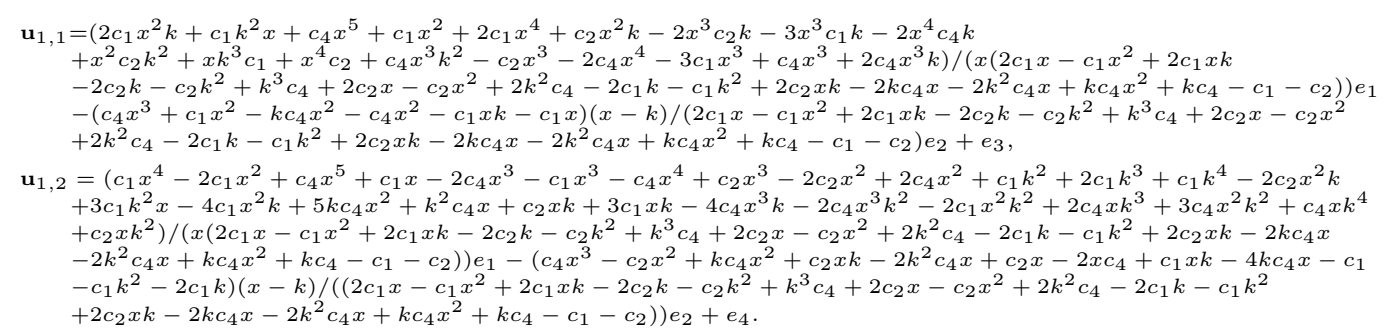

The submodules given by the last three solutions of the $c_{i}$ 's are more complicated (see [25, Example 4.4.1]).

\subsection{Eigenrings and decomposition of Laurent-Ore modules}

We discuss another approach to factoring Laurent-Ore modules, which is not based on the associated equations method. This method is first introduced in [23] to factor linear ordinary differential operators using eigenrings of the operators. Three algorithms are presented there for computing eigenrings. Significant improvements on these algorithms are described in $[4,10]$. Although the eigenring method does not always factor reducible operators, it often yields factors quickly. This method has been generalized in $[1,5]$ for systems of linear difference equations, and in [3] recently for systems of linear partial differential equations in positive characteristic. We will generalize this method for factoring Laurent-Ore modules.

Let $R$ be an arbitrary ring and $M$ be an $R$-module. Recall that $\operatorname{End}_{R}(M)$ is the set of all $R$-linear maps on $M$. Clearly, $\operatorname{End}_{R}(M)$ becomes a ring with the usual addition and the composition of maps adopted as the multiplication.

Definition 6.5 Let $M$ be an $R$-module. A set of elements $\pi_{1}, \ldots, \pi_{s}$ of $\operatorname{End}_{R}(M)$ is called a set of orthogonal idempotents if they satisfy

$$
\sum_{i=1}^{s} \pi_{i}=\mathbf{1} \quad \text { and } \quad \pi_{i} \pi_{j}=\mathbf{0} \quad \text { whenever } i \neq j,
$$

where $\mathbf{1}$ and $\mathbf{0}$ are the identity map and the zero map on $M$, respectively. 
Although it is not stated in Definition 6.5, the maps $\pi_{i}$ are all idempotent. Indeed, the condition (5) implies that $\pi_{i}^{2}=\sum_{j=1}^{s} \pi_{i} \pi_{j}=\pi_{i}\left(\sum_{j=1}^{s} \pi_{j}\right)=\pi_{i}$ for each $i$.

It is stated in Exercise 7 of $[12,($ Chapter $1, \S 1)]$ that

Proposition 6.6 Let $M$ be an R-module. If $\operatorname{End}_{R}(M)$ has a set of orthogonal idempotents $\pi_{1}, \ldots, \pi_{s}$ then $M=\oplus_{i=1}^{s} \pi_{i}(M)$. Conversely, if $M$ can be written as a direct sum of submodules $M=N_{1} \oplus \cdots \oplus N_{s}$ then $\left\{\pi_{1}, \ldots, \pi_{s}\right\}$ is a set of orthogonal idempotents of $\operatorname{End}_{R}(M)$ where $\pi_{i}$ is the projection from $M$ to $N_{i}$.

For any $R$-module $M, \operatorname{End}_{R}(M)$ always has a set of orthogonal idempotents $\{\mathbf{0}, \mathbf{1}\}$, which is called the trivial orthogonal idempotents of $\operatorname{End}_{R}(M)$. As a direct consequence of Proposition 6.6, an $R$-module $M$ is decomposable if and only if $\operatorname{End}_{R}(M)$ contains a nontrivial set of orthogonal idempotents.

Let $F$ be a $\Delta$-field, $C$ its field of constants and $\mathcal{L}=F\left[\partial_{1}, \ldots, \partial_{m}, \partial_{\ell+1}^{-1}, \ldots, \partial_{m}^{-1}\right]$ the Laurent-Ore algebra over $F$. Here we do not assume that $C$ is algebraically closed.

For an $\mathcal{L}$-module $M$, the endomorphism ring $\operatorname{End}_{\mathcal{L}}(M)$ is called the eigenring of $M$ and denoted $\mathcal{E}(M)$. Then a map $\phi \in \operatorname{End}_{F}(M)$ belongs to $\mathcal{E}(M)$ if and only if $\phi$ commutes with the $\partial_{i}$ and $\partial_{j}^{-1}$ for all $i, j$ with $1 \leq i \leq m$ and $\ell+1 \leq j \leq m$. However, since $M$ is an $\mathcal{L}$-module on which the $\partial_{j}^{-1}$ act, the commutativity of $\phi$ with the $\partial_{j}$ for $\ell+1 \leq j \leq m$ implies $\partial_{j} \circ \phi \circ \partial_{j}^{-1}(\mathbf{w})=\phi(\mathbf{w})$ and further $\phi \circ \partial_{j}^{-1}(\mathbf{w})=\partial_{j}^{-1} \circ \phi(\mathbf{w})$ for $\mathbf{w} \in M$. Hence, $\phi \in$ $\operatorname{End}_{F}(M)$ belongs to $\mathcal{E}(M)$ if and only if $\phi$ commutes with all the $\partial_{i}$ for $1 \leq i \leq m$.

Let $M$ be a Laurent-Ore module with an $F$-basis $\left\{\mathbf{e}_{1}, \ldots, \mathbf{e}_{n}\right\}$. Suppose that

$$
\partial_{i}\left(\mathbf{e}_{1}, \ldots, \mathbf{e}_{n}\right)^{\tau}=B_{i}\left(\mathbf{e}_{1}, \ldots, \mathbf{e}_{n}\right)^{\tau}, \quad i=1, \ldots, m,
$$

where $B_{i} \in F^{n \times n}$ for $1 \leq i \leq m$ and the $B_{j}$ are invertible for $j>\ell$. In practice, the maps in $\mathcal{E}(M)$ can be interpreted in terms of the $B_{i}$. Let $\phi \in \operatorname{End}_{F}(M)$ and $P \in F^{n \times n}$ be its transformation matrix given by $\left(\phi\left(\mathbf{e}_{1}\right), \ldots, \phi\left(\mathbf{e}_{n}\right)\right)^{\tau}=P\left(\mathbf{e}_{1}, \ldots, \mathbf{e}_{n}\right)^{\tau}$. Let $\mathbf{w}=\sum_{i=1}^{n} a_{i} \mathbf{e}_{i} \in M$ where $a_{i} \in F$. Then

$$
\phi(\mathbf{w})=\sum_{i=1}^{n} a_{i} \phi\left(\mathbf{e}_{i}\right)=\left(a_{1}, \ldots, a_{n}\right)\left(\phi\left(\mathbf{e}_{1}\right), \ldots, \phi\left(\mathbf{e}_{n}\right)\right)^{\tau}=\left(a_{1}, \ldots, a_{n}\right) P\left(\mathbf{e}_{1}, \ldots, \mathbf{e}_{n}\right)^{\tau} .
$$

One can verify that the conditions $\partial_{i}(\phi(\mathbf{w}))=\phi\left(\partial_{i}(\mathbf{w})\right)$ hold for $\mathbf{w} \in M$ and $1 \leq i \leq m$ if and only if $\delta_{i}(P)=B_{i} P-P B_{i}$ for $i \leq \ell$ and $\sigma_{j}(P)=B_{j} P B_{j}^{-1}$ for $j>\ell$. Hence the eigenring $\mathcal{E}(M)$ can be defined equivalently to be

$$
\mathcal{E}(M)=\left\{P \in F^{n \times n} \mid \delta_{i}(P)=B_{i} P-P B_{i} \text { for } i \leq \ell \text { and } \sigma_{j}(P)=B_{j} P B_{j}^{-1} \text { for } j>\ell\right\} .
$$

Clearly, the identity matrix $\mathbf{1}_{n} \in \mathcal{E}(M)$ and $\mathcal{E}(M)$ is a $C$-subalgebra of $F^{n \times n}$ of dimension not greater than $n^{2}$. Moreover, $C \cdot \mathbf{1}_{n} \subseteq \mathcal{E}(M)$ where $C \cdot \mathbf{1}_{n}$ denotes the set of all matrices of the form $c \cdot \mathbf{1}_{n}$ where $c \in C$.

As a natural generalization of the results in [2], [20, Proposition 2.13] or [23] for the case of linear ordinary differential equations, we have

Theorem 6.7 Let $M$ be an $\mathcal{L}$-module of dimension $n$. Then 
(i) If $\mathcal{E}(M) \neq C \cdot \mathbf{1}_{n}$ then $M$ is reducible.

(ii) If $M$ is decomposable then $\mathcal{E}(M) \neq C \cdot \mathbf{1}_{n}$.

(iii) If $M$ is completely reducible, then $M$ is irreducible if and only if $\mathcal{E}(M)=C \cdot \mathbf{1}_{n}$.

Given a Laurent-Ore module $M$ of dimension $n$, we now use the formula (6) to compute $\mathcal{E}(M)$. Let $P \in \mathcal{E}(M)$ be a matrix of $n^{2}$ indeterminates $z_{i j}$. From (6), we get a system $\partial_{i}(\mathbf{z})=A_{i} \mathbf{z}$ where $A_{i} \in F^{n^{2} \times n^{2}}$ and $\mathbf{z}=\left(z_{11}, \ldots, z_{1 n}, \ldots, z_{n 1}, \ldots, z_{n n}\right)^{\tau}$. This system is clearly $\partial$-finite, so its rational solutions can be found by a specialized version of the method in [15]. A $C$-basis of all rational solutions of this system yields a $C$-basis $\left\{P_{1}, \ldots, P_{r}\right\}$ of all rational solutions of $\mathcal{E}(M)$. Without loss of generality, we assume that $P_{1}=\mathbf{1}_{n}$. Therefore $\mathcal{E}(M)=\oplus_{i=1}^{r} C \cdot P_{i}$. If $r=1$, then $\mathcal{E}(M)$ is trivial and $M$ is indecomposable by Theorem 6.7 (ii). Otherwise, each eigenvalue $\lambda$ of a nontrivial $P \in \mathcal{E}(M)$ will produce a submodule $\{\mathbf{w} \in M \mid P \mathbf{w}=\lambda \mathbf{w}\}$ of $M$. If $\mathcal{E}(M)$ has a set of nontrivial orthogonal idempotents $\pi_{1}, \ldots, \pi_{s}$, then we derive a decomposition $M=\pi_{1}(M) \oplus \cdots \oplus \pi_{s}(M)$. If $M$ is furthermore completely reducible, a maximal decomposition of $M$ can be obtained by applying the eigenring method recursively on the submodules in the above decomposition.

Example 6.8 Let $F, \delta_{x}, \sigma_{k}$ be as given in Example 3.2, $\mathcal{L}=F\left[\partial_{x}, \partial_{k}, \partial_{k}^{-1}\right]$ the LaurentOre algebra and $M$ an $\mathcal{L}$-module of dimension two. Suppose that $\left\{\mathbf{e}_{1}, \mathbf{e}_{2}\right\}$ is a basis of $M$ satisfying $\partial_{x}\left(\mathbf{e}_{1}, \mathbf{e}_{2}\right)^{\tau}=B_{x}\left(\mathbf{e}_{1}, \mathbf{e}_{2}\right)^{\tau}$ and $\partial_{k}\left(\mathbf{e}_{1}, \mathbf{e}_{2}\right)^{\tau}=B_{k}\left(\mathbf{e}_{1}, \mathbf{e}_{2}\right)^{\tau}$ where $B_{x}=\left(\begin{array}{cc}1 & 0 \\ 0 & 0\end{array}\right)$ and $B_{k}=\left(\begin{array}{cc}1 & 0 \\ 0 & k\end{array}\right)$.

We now compute the eigenring of $M$. Let $P \in \mathcal{E}(M)$ be a $2 \times 2$ matrix with indeterminate entries $z_{11}, z_{12}, z_{21}$ and $z_{22}$. The conditions $\delta_{x}(P)=B_{x} P-P B_{x}$ and $\sigma_{k}(P)=B_{k} P B_{k}^{-1}$ yields a system $\mathcal{A}:\left\{\delta_{x}(\mathbf{z})=A_{x} \mathbf{z}, \sigma_{k}(\mathbf{z})=A_{k} \mathbf{z}\right\}$ where $\mathbf{z}=\left(z_{11}, z_{12}, z_{21}, z_{22}\right)^{\tau}$,

$$
A_{x}=\left(\begin{array}{cccc}
0 & \frac{k\left(-x+x^{2}-k x+2 k\right)}{(x-k)(x-1)} & \frac{x^{2}-k x+3 k-2 x}{x(x-k) k(x-1)} & 0 \\
-\frac{x^{2}-k x+3 k-2 x}{x(x-k) k(x-1)} & -\frac{x^{3}-k x^{2}-2 x+3 k+k x}{x(x-k)(x-1)} & 0 & \frac{x^{2}-k x+3 k-2 x}{x(x-k) k(x-1)} \\
-\frac{k\left(-x+x^{2}-k x+2 k\right)}{(x-k)(x-1)} & 0 & \frac{x^{3}-k x^{2}-2 x+3 k+k x}{x(x-k)(x-1)} & \frac{k\left(-x+x^{2}-k x+2 k\right)}{(x-k)(x-1)} \\
0 & -\frac{k\left(-x+x^{2}-k x+2 k\right)}{(x-k)(x-1)} & -\frac{x^{2}-k x+3 k-2 x}{x(x-k) k(x-1)} & 0
\end{array}\right)
$$

and

$$
A_{k}=\frac{1}{\gamma}\left(\begin{array}{cccc}
\frac{x^{2}-2 k x-x+k^{2}}{k} \alpha & -x \alpha \beta & -\frac{x^{2}-2 k x-x+k^{2}}{k^{2}} \beta & \frac{x}{k} \beta^{2} \\
\frac{1}{k(k+1)} \alpha \beta & \frac{1}{k+1} \alpha^{2} & -\frac{1}{k^{2}(k+1)} \beta^{2} & -\frac{1}{k(k+1)} \alpha \beta \\
\frac{x(k+1)\left(x^{2}-2 k x-x+k^{2}\right)}{k} \beta & -(k+1) x^{2} \beta^{2} & \frac{(k+1)\left(x^{2}-2 k x-x+k^{2}\right)^{2}}{k^{2}} & \frac{x(k+1)\left(2 k x+x-x^{2}-k^{2}\right)}{k} \beta \\
\frac{x}{k} \beta^{2} & x \alpha \beta & \frac{x^{2}-2 k x-x+k^{2}}{k^{2}} \beta & \frac{x^{2}-2 k x-x+k^{2}}{k} \alpha,
\end{array}\right),
$$

with $\alpha=k+1+k x^{2}-k^{2} x-x, \beta=k+1+k x-k^{2}-x$ and $\gamma=(x-k)(x-k-1)(x-1)^{2}$. All rational solutions of $\mathcal{A}$ are of the form

$$
c_{1}\left(\frac{1}{x-1},-\frac{1}{k(x-1)}, \frac{x k}{x-1},-\frac{x}{x-1}\right)+c_{2}\left(-\frac{x}{x-1}, \frac{1}{k(x-1)},-\frac{x k}{x-1}, \frac{1}{x-1}\right),
$$


for $c_{1}, c_{2} \in \mathbb{C}$. So

$$
\begin{aligned}
\mathcal{E}(M) & =\left\{\left(\begin{array}{cc}
\frac{c_{1}-c_{2} x}{x-1} & \frac{c_{2}-c_{1}}{k(x-1)} \\
\frac{\left(c_{1}-c_{2}\right) x k}{x-1} & \frac{c_{2}-c_{1} x}{x-1}
\end{array}\right), \text { for any } c_{1}, c_{2} \in \mathbb{C}\right\} \\
& =\mathbb{C}\left(\begin{array}{cc}
\frac{1}{x-1} & -\frac{1}{k(x-1)} \\
\frac{k x}{x-1} & -\frac{x}{x-1}
\end{array}\right) \oplus \mathbb{C}\left(\begin{array}{cc}
-\frac{x}{x-1} & \frac{1}{k(x-1)} \\
-\frac{k x}{x-1} & \frac{1}{x-1}
\end{array}\right) .
\end{aligned}
$$

Recall that the necessary condition for $\left\{P_{1}, \ldots, P_{s}\right\} \subset \mathcal{E}(M)$ being a set of orthogonal idempotents is that $P_{i}^{2}=P_{i}$ for each $i$. Substitute

$$
P=\left(\begin{array}{cc}
\frac{c_{1}-c_{2} x}{x-1} & \frac{c_{2}-c_{1}}{k(x-1)} \\
\frac{\left(c_{1}-c_{2}\right) x k}{x-1} & \frac{c_{2}-c_{1} x}{x-1}
\end{array}\right)
$$

into the relation $P^{2}=P$, we obtain three solutions:

$$
P_{0}=\mathbf{1}_{2}, \quad P_{1}=\left(\begin{array}{cc}
-\frac{1}{x-1} & \frac{1}{k(x-1)} \\
-\frac{k x}{x-1} & \frac{x}{x-1}
\end{array}\right), \quad P_{2}=\left(\begin{array}{cc}
\frac{x}{x-1} & -\frac{1}{k(x-1)} \\
\frac{k x}{x-1} & -\frac{1}{x-1}
\end{array}\right) .
$$

Among which, we find $P_{1} P_{2}=0$ and $P_{1}+P_{2}=\mathbf{1}_{2}$. So $\left\{P_{1}, P_{2}\right\}$ is a set of nontrivial orthogonal idempotents of $\mathcal{E}(M)$. We have

$$
P_{1}(M)=\left\{P_{1}(\mathbf{w}) \mid \mathbf{w} \in M\right\}=\left\{\left(a_{1}, a_{2}\right) P_{1}\left(\mathbf{e}_{1}, \mathbf{e}_{2}\right)^{\tau} \mid a_{1}, a_{2} \in F\right\}=F \cdot\left(\mathbf{e}_{1}-\frac{1}{k} \mathbf{e}_{2}\right)
$$

and

$$
P_{2}(M)=\left\{P_{2}(\mathbf{w}) \mid \mathbf{w} \in M\right\}=\left\{\left(a_{1}, a_{2}\right) P_{2}\left(\mathbf{e}_{1}, \mathbf{e}_{2}\right)^{\tau} \mid a_{1}, a_{2} \in F\right\}=F \cdot\left(\mathbf{e}_{1}-\frac{1}{k x} \mathbf{e}_{2}\right) .
$$

Therefore, $P_{1}(M) \oplus P_{2}(M)$ is a decomposition of $M$ into two nontrivial submodules.

The eigenring method, however, may fail to find any factor of a Laurent-Ore module even this module is reducible. This happens when the eigenring of that module is trivial.

\section{Concluding remarks}

In this paper we have discussed how to solve and factor $\partial$-finite systems. A key technique described here is to use the notion of modules of formal solutions to connect $\partial$-finite systems with fully integrable systems, while the latter systems are very similar to linear ordinary differential (difference) equations. This technique naturally gives rise to PicardVessiot extensions for $\partial$-finite systems. Since Picard-Vessiot extensions are a stepping-stone to introduce Galois groups, it would be interesting to extend (part of) the Galois theory for linear ordinary (difference) equations to $\partial$-finite systems. We presented some methods for determining linear dimension of a linear functional system. We also generalized Beke's method and the eigenring approach to factor Laurent-Ore modules. The work on factoring Laurent-Ore modules is however preliminary, because efficiency and applications of these two methods have not yet been considered. 


\section{References}

[1] Barkatou, M.A., Rational solutions of matrix difference equations: the problem of equivalence and factorization, Proc. ISSAC1999, 277-282, ACM Press, 1999.

[2] Barkatou, M.A., On the reduction of matrix pseudo-linear equations, Technical report, Rapport de Recherche de l'institut IMAG, 2001.

[3] Barkatou, M.A., Cluzeau, T., Weil, J.-A., Factoring partial differential systems in positive characteristic, Differential Equations and Symbolic Computation, chapter 13, Birkhauser, Basel Boston, 2005.

[4] Barkatou, M.A., Pflügel, E., On the equivalence problem of linear differential systems and its application for factoring completely reducible systems, Proc. ISSAC1998, 268-275, ACM Press, 1998.

[5] Bomboy, R., Réductibilité et Résolubilité des Équations aux Différences Finies, PhD thesis, Université de Nice-Sophia Antipolis, 2001.

[6] Bronstein, M., Li., Z., Wu, M., Picard-Vessiot extensions for linear functional systems, Proc. ISSAC2005, 68-75, ACM Press, 2005.

[7] Chyzak, F., Quadrat, A., Robertz, D., Oremodules: A symbolic package for the study of multidimensional linear systems, Proc. MTNS2004, Leuven (Belgium), CDRom, 2004.

[8] Chyzak, F., Salvy, B., Non-commutative elimination in Ore algebras proves multivariate identities, J. of Symbolic Computation, 26(2):187-228, August 1998.

[9] Compoint, E., Weil, J.-A., Absolute reducibility of differential operators and Galois groups, J. of Algebra, 275(1):77-105, 2003.

[10] van Hoeij, M., Rational solutions of the mixed differential equation and its application to factorization of differential operators, Proc. ISSAC1996, 219-225, ACM Press, 1996.

[11] Labahn, G., Li, Z., Hyperexponential solutions of finite-rank ideals in orthogonal Ore algebras, Proc. ISSAC2004, 213-220, ACM Press, 2004.

[12] Lam, T. Y., A First Course in Non-Commutative Rings, Graduate Texts in Mathematics, volume 131, Springer-Verlag, 1991.

[13] Li, Z., Schwarz, F., Tsarev, S., Factoring zero-dimensional ideals of linear partial differential operators, Proc. ISSAC2002, 168-175, ACM Press, 2002.

[14] Li, Z., Schwarz, F., Tsarev, S., Factoring systems of linear PDE's with finite-dimensional solution spaces, J. of Symbolic Computation, 36:443-471, 2003.

[15] Li, Z., Singer, M.F., Wu, M., Zheng, D., A recursive method for determining the one-dimensional submodules of Laurent-Ore modules, Proc. of ISSAC2006, 220-227, ACM Press, 2006.

[16] Malgrange, B., Motivations and introduction to the theory of D-modules, Computer Algebra and Differential Equations, volume 193 of LMS Lecture Note Series, 3-20, Cambridge University Press, 1994.

[17] Marcus, M., Finite Dimensional Multilinear Algebra, Part II, Marcel Dekker, New York, 1975.

[18] Pauer, F., Unterkircher, A., Gröbner bases for ideals in Laurent polynomial rings and their applications to systems of difference equations, Proc. AAECC1999, volume 9, 271-291, 1999.

[19] van der Put, M., Singer, M.F., Galois Theory of Difference Equations, Lecture Notes in Mathematics 1666, Springer, 1997.

[20] van der Put, M., Singer, M.F., Galois Theory of Linear Differential Equations, Grundlehren der Mathematischen Wissenschaften, volume 328, Springer, Heidelberg, 2003.

[21] Rotman, J. J., An Introduction to Homological Algebra, Academic Press, New York, 1979.

[22] Rowen, L.H., Ring Theory, I, San Diego, CA:Academic Press, 1988. 
[23] Singer, M.F., Testing reducibility of linear differential operators: a group theoretic perspective, J. of Appl. Alg. in Eng. Comm. and Compu., 7(2):77-104, 1996.

[24] Zhou, M., Winkler, F., Gröbner bases in difference-differential modules, Proc. ISSAC2006, 353360, ACM Press, 2006.

[25] Wu, M., On Solutions of Linear Functional Systems and Factorization of Modules over LaurentOre Algebras, PhD thesis, Chinese Academy of Sciences and Université de Nice, 2005.

[26] Zampieri, S., A solution of the Cauchy problem for multidimensional discrete linear shiftinvariant systems, Linear Algebra and Its Applications, 202:143-162, 1994. 